\title{
LARGE AUTOMORPHISM GROUPS OF HYPERELLIPTIC KLEIN SURFACES
}

\author{
E. BUJALANCE AND J. J. ETAYO \\ (Communicated by Irwin Kra)
}

\begin{abstract}
In this paper we study the groups of automorphisms of all hyperelliptic bordered Klein surfaces of genus $p>3$ having at least $4(p-1)$ automorphisms.
\end{abstract}

1. Introduction. We mean as Klein surface a compact surface with a dianalytic structure (see [1]). An automorphism of the surface $X$ is a dianalytic homeomorphism $f: X \rightarrow X$.

A bordered surface $X$ is called hyperelliptic if it admits an automorphism $\varphi$ of order two such that the quotient $X / \varphi$ has algebraic genus zero (see $[4$, p. 144]).

Bordered Klein surfaces of algebraic genus $p$ have at most $12(p-1)$ automorphisms [9, Theorem 5]. In this paper we study the groups of automorphisms of all hyperelliptic surfaces of genus $p>3$ having at least $4(p-1)$ automorphisms. In case $p=2$ or $p=3$, all possible automorphism groups have been obtained in [6 and 5]. In particular, for $p=2, D_{4}$ and $D_{6}$ are cases of order $>4$ : for $p=3, D_{6}$ and $Z_{2} \times D_{4}$ are the groups of order $>8$.

2. Preliminaries. The main tool in our study is the theory of non-Euclidean crystallographic (NEC) groups. An NEC group is a discrete subgroup of the isometries group of the hyperbolic plane, including orientation-reversing elements, with compact quotient space.

NEC groups are classified by their signature, that is

$$
\left(g, \pm,\left[m_{1}, \ldots, m_{r}\right],\left\{\left(n_{11}, \ldots, n_{1 s_{1}}\right), \ldots,\left(n_{k 1}, \ldots, n_{k s_{k}}\right)\right\}\right) \text {. }
$$

The $m_{i}$ 's are the proper periods, the $\left(n_{i 1}, \ldots, n_{i s_{i}}\right)$ are the period-cycles and the $n_{i j}$ the periods of the period-cycles. The signature determines a presentation of the group, given by generators

(i) $x_{i}, i=1, \ldots, r$,

(ii) $e_{i}, i=1, \ldots, k$,

(iii) $c_{i j}, i=1, \ldots, k, j=0, \ldots, s_{i}$,

(iv) (if sign ' + ') $a_{i}, b_{i}, i=1, \ldots, g$,

$$
\text { (if sign' }- \text { ') } d_{i}, i=1, \ldots, g \text {, }
$$

and relations

(i) $x_{i}^{m_{i}}=1, i=1, \ldots, r$,

Received by the editors April 18, 1986 and, in revised form, April 1, 1987.

1980 Mathematics Subject Classification (1985 Revision). Primary 14H45; Secondary 30F99.

This work was presented at Groups-St. Andrews, July 1985, sponsored by the London Mathematical Society and the Edinburgh Mathematical Society.

Partially supported by "Comisión Asesora de Investigación Científica y Técnica". 
(ii) $e_{i}^{-1} c_{i 0} e_{i} c_{i s_{i}}=1, i=1, \ldots, k$,

(iii) $c_{i, j-1}^{2}=c_{i j}^{2}=\left(c_{i, j-1} c_{i j}\right)^{n_{i j}}=1, i=1, \ldots, k, j=1, \ldots, s_{i}$,

(iv) (if sign ' + ') $x_{1} \cdots x_{r} e_{1} \cdots e_{k} a_{1} b_{1} a_{1}^{-1} b_{1}^{-1} \cdots a_{g} b_{g} a_{g}^{-1} b_{g}^{-1}=1$, (if sign ' -') $x_{1} \cdots x_{r} e_{1} \cdots e_{k} d_{1}^{2} \cdots d_{g}^{2}=1$.

An NEC group $\Gamma$ with signature $(*)$ has an associated area

$$
|\Gamma|=2 \pi\left(\alpha g+k-2+\sum_{i=1}^{r}\left(1-\frac{1}{m_{i}}\right)+\frac{1}{2} \sum_{i=1}^{k} \sum_{j=1}^{s_{i}}\left(1-\frac{1}{n_{i j}}\right)\right),
$$

$\alpha=1$ if the sign is ' - ' and 2 if the sign is ' + '.

A Klein surface $X$ of topological genus $g$ with $k$ boundary components may be expressed as $D / \Gamma, D$ being the hyperbolic plane and $\Gamma$ an NEC group with signature $\left(g, \pm,[-],\left\{(-), .{ }^{k} .,(-)\right\}\right)$, the sign being ' + ' if $X$ is orientable, and '-' if nonorientable. The relation between algebraic and topological genera, and number of boundary components, is $p=\alpha g+k-1$.

If $G$ is a group of automorphisms of $D / \Gamma$, it may be expressed as $\Gamma^{\prime} / \Gamma$, where $\Gamma^{\prime}$ is an NEC group of which $\Gamma$ is a normal subgroup. Then the order of $G,|G|$, is $|\Gamma| /\left|\Gamma^{\prime}\right|$. Hence, if $|G| \geq 4(p-1)$, one obtains by a simple calculation, keeping in mind that if $\Gamma^{\prime}$ has a nonempty period-cycle, this has at least two consecutive periods equal to two $[7, \S 4]$ that $\Gamma^{\prime}$ has one of the following signatures:

$$
\begin{aligned}
& \text { (i) }(0,+,[-],\{(2,2,2, n)\}) \text {, } \\
& \text { (ii) }(0,+,[-],\{(2,2,3,3)\}) \text {, } \\
& \text { (iii) }(0,+,[3],\{(2,2)\}) \text {, } \\
& \text { (iv) }(0,+,[2,3],\{(-)\}) \text {, } \\
& \text { (v) }(0,+,[-],\{(2,2,3,4)\}) \text {, } \\
& |G|=\frac{4 n}{n-2}(p-1), \\
& |G|=6(p-1), \\
& \text { (vi) }(0,+,[-],\{(2,2,3,5)\}) \text {, } \\
& \text { (vii) }(0,+,[-],\{(2,2,2,2,2)\}) \text {, } \\
& \text { (viii) }(0,+,[2],\{(2,2,2)\}) \text {, } \\
& \text { (ix) }(0,+,[-],\{(2,2,4,4)\}) \text {, } \\
& \text { (x) }(0,+,[4],\{(2,2)\}) \text {, } \\
& \text { (xi) }(0,+,[2,4],\{(-)\}) \text {, } \\
& \text { (xii) }(0,+,[-],\{(2,2,3,6)\}) \text {, } \\
& |G|=\frac{24}{5}(p-1), \\
& |G|=\frac{30}{7}(p-1) \text {, } \\
& |G|=4(p-1),
\end{aligned}
$$

(This list, for groups of order $\geq 4 p$, may be deduced from [9].)

3. Large automorphism groups. In [4, Theorem 4.5] hyperelliptic Klein surfaces have been characterized in the following way.

Let $\Gamma$ be an NEC group with signature $\left(g, \pm,[-],\left\{(-), .{ }^{k},(-)\right\}\right)$. Then $D / \Gamma$ is hyperelliptic if and only if there exists a unique NEC group $\Gamma_{1}$ with $\left|\Gamma_{1}: \Gamma\right|=2$, and whose signature is

(1) $(0,+,[-],\{(2, .2 k, 2)\})$ if $g=0$,

(2) $\left(0,+,\left[2,{ }^{2 g+k}, 2\right],\{(-)\}\right)$ if $g \neq 0$ and $\Gamma$ has sign ' + ' (and then $k<3$ ),

(3) $(0,+,[2, .9 ., 2],\{(2, .2 k ., 2)\})$ if $\Gamma$ has sign ' - '.

When $G=\Gamma^{\prime} / \Gamma$ is the automorphism group of the hyperelliptic surface $D / \Gamma$ then $\Gamma_{1} \triangleleft \Gamma^{\prime}$. We call $G_{1}=\Gamma^{\prime} / \Gamma_{1}$, and it is cyclic or dihedral, since it is the automorphism group of the disc $D / \Gamma_{1}$. 
In this section we obtain all hyperelliptic surfaces of genus $p>3$ with at least $4(p-1)$ automorphisms. It was proven in [3] that no such surface has $12(p-1)$ automorphisms.

Now we state three technical lemmas.

LEMMA 1. If $\Gamma^{\prime}$ has signature (ii), (iii) or (iv), and there exists a normal subgroup $\Gamma$ of $\Gamma^{\prime}$ having signature $(g, \pm,[-],\{(-), . k .,(-)\}), D / \Gamma$ being a hyperelliptic surface of algebraic genus $p$, such that $\Gamma^{\prime} / \Gamma$ is the automorphism group of $D / \Gamma$ having order $6(p-1)$, then $p \leq 2$.

Proof. Let $\Gamma^{\prime}$ be a group with signature (ii). If there exists $\Gamma$ in the above conditions, there is an epimorphism $\theta_{1}: \Gamma^{\prime} \rightarrow G_{1}$ such that $\operatorname{ker} \theta_{1}=\Gamma_{1}$, the group of the hyperellipticity of $D / \Gamma$. If $\Gamma_{1}$ had signature (1), $\theta_{1}$ should be defined by $\theta_{1}\left(c_{10}\right)=x, \theta_{1}\left(c_{11}\right)=1, \theta_{1}\left(c_{12}\right)=1, \theta_{1}\left(c_{13}\right)=y, \theta_{1}\left(c_{14}\right)=x$, which is impossible because $c_{12} c_{13}$ has order three: if $\Gamma_{1}$ had signature (2) or (3) we may not obtain proper periods equal to two in $\operatorname{ker} \theta_{1}$. Hence $\theta_{1}$ exists in no case.

If $\Gamma^{\prime}$ has signature (iii), and $\Gamma_{1}$ has signature $(1)$, then $\theta_{1}\left(c_{10}\right)=1, \theta_{1}\left(c_{11}\right)=1$, $\theta_{1}\left(c_{12}\right)=1, \theta_{1}\left(e_{1}\right)=x, \theta_{1}\left(x_{1}\right)=x^{-1} ;$ as $\left|G_{1}\right|=3(p-1)$, we have $3(p-1)=3$, and so $p=2$. If $\Gamma_{1}$ has signature (2) we may not obtain simultaneously proper periods and period-cycles, and so $\theta_{1}$ does not exist, and the same holds in case $\Gamma_{1}$ has signature (3).

If $\Gamma^{\prime}$ has signature (iv), $\Gamma_{1}$ may not have signature (1) or (3) because it has no nonempty period-cycles. If $\Gamma_{1}$ has signature $(2)$, then $\theta_{1}\left(x_{1}\right)=1, \theta_{1}\left(x_{2}\right)=x$, $\theta_{1}\left(e_{1}\right)=x^{-1}, \theta_{1}\left(c_{10}\right)=1$. Then $3(p-1)=3$, and so $p=2$.

LEMMA 2. If $\Gamma^{\prime}$ has signature (v) (respectively (vi)), there exists no normal subgroup $\Gamma$ of $\Gamma^{\prime}$ having signature $(g, \pm,[-],\{(-), . . .,(-)\}), D / \Gamma$ being a hyperelliptic surface of algebraic genus $p$, such that $\Gamma^{\prime} / \Gamma$ is the automorphism group of $D / \Gamma$ having order $\frac{24}{5}(p-1)$ (respectively $\left.\frac{30}{7}(p-1)\right)$.

PROOF. If there exists $\Gamma$ in the above conditions we may define an epimorphism $\theta_{1}: \Gamma^{\prime} \rightarrow G_{1}, G_{1}$ having order $\frac{12}{5}(p-1)$ (respectively $\frac{15}{7}(p-1)$ ) with kernel $\Gamma_{1}$ having signature $(1),(2)$ or $(3)$.

If $\Gamma_{1}$ has signature $(1), \theta_{1}$ is given by $\theta_{1}\left(c_{10}\right)=1, \theta_{1}\left(c_{11}\right)=1, \theta_{1}\left(c_{12}\right)=x$, $\theta_{1}\left(c_{13}\right)=y, \theta_{1}\left(c_{14}\right)=1$. This possibility holds only for case $(\mathrm{v})$, and then $G_{1}$ is generated by $x, y$, satisfying $x^{2}=y^{2}=(x y)^{3}=1$. Hence $\left|G_{1}\right|=6=\frac{12}{5}(p-1)$, and $p$ is not an integer.

When $\Gamma_{1}$ has signature (2), the epimorphism $\theta_{1}$ is defined by $\theta_{1}\left(c_{10}\right)=x$, $\theta_{1}\left(c_{11}\right)=1, \theta_{1}\left(c_{12}\right)=y, \theta_{1}\left(c_{13}\right)=z, \theta_{1}\left(c_{14}\right)=x$, where $x z$ has order 4 (respectively 5$), y z$ has order 3 , and so $y \neq 1$. Then the number of proper periods of ker $\theta_{1}, p+1$, equals $\frac{12}{5}(p-1) / 4$ (respectively $\left.\frac{15}{7}(p-1) / 5\right)$ [2, Theorem 2]. Hence $\frac{3}{5}(p-1)=p+1\left(\right.$ respectively $\left.\frac{3}{7}(p-1)=p+1\right)$, impossible.

Finally, one may not get an epimorphism $\theta_{1}$ with kernel having signature (3).

LEMMA 3. If $\Gamma^{\prime}$ has signature (vii), (viii), (ix), (x), (xi) or (xii) and there exists a normal subgroup $\Gamma$ of $\Gamma^{\prime}$ having signature $(g, \pm,[-],\{(-), . k .,(-)\}), D / \Gamma$ being a hyperelliptic surface of algebraic genus $p$, such that $\Gamma^{\prime} / \Gamma$ is the automorphism group of $D / \Gamma$ having order $4(p-1)$, then $p \leq 3$.

ProOF. Let $\Gamma^{\prime}$ have signature (vii). Then we have an epimorphism $\theta_{1}$ from $\Gamma^{\prime}$ onto $G_{1}$ with kernel $\Gamma_{1}$. If $\Gamma_{1}$ has signature (1), $\theta_{1}$ is defined by $\theta_{1}\left(c_{10}\right)=x$, 
$\theta_{1}\left(c_{11}\right)=1, \theta_{1}\left(c_{12}\right)=1, \theta_{1}\left(c_{13}\right)=y, \theta_{1}\left(c_{14}\right)=z, \theta_{1}\left(c_{15}\right)=x$; if $y=z=1$, then $G_{1}=Z_{2}$, and so $2(p-1)=2, p=2$; if $y=1, z \neq 1$, then we call $r$ the order of $x z$ and the number of period-cycles of $\operatorname{ker} \theta_{1}, 1$, equals $2(p-1) / 2 r[7, \S 3]$; so $r=p-1$; and the number of periods of the period-cycle, $2 k$, equals $4 r[7]$, and so $2 r=k$. Hence $2(p-1)=k$ and $p=k / 2+1$; since in case (1) $p=k-1$, we obtain $p=3$. Finally, if $y \neq 1 \neq z$, calling again $r$ the order of $x z$, we obtain $r=p-1$, and the number of periods of the period-cycle, $2 k$, is now $2 r$. Hence $2 k=2 r$, and so $p+1=p-1$, impossible. For any other election of $y$ and $z$, the kernel of $\theta_{1}$ would not have signature (1).

If $\Gamma_{1}$ has signature (2), the epimorphism $\theta_{1}$ is given by $\theta_{1}\left(c_{10}\right)=x, \theta_{1}\left(c_{11}\right)=1$, $\theta_{1}\left(c_{12}\right)=y, \theta_{1}\left(c_{13}\right)=z, \theta_{1}\left(c_{14}\right)=t, \theta_{1}\left(c_{15}\right)=x$. Then the number of proper periods of $\operatorname{ker} \theta_{1}$ is $\beta(p-1)$, that must equal $p+1$, and so $p=2$ or $p=3$.

When $\Gamma_{1}$ has signature (3), $\theta_{1}$ is defined by $\theta_{1}\left(c_{10}\right)=x, \theta_{1}\left(c_{11}\right)=1, \theta_{1}\left(c_{12}\right)=1$, $\theta_{1}\left(c_{13}\right)=y, \theta_{1}\left(c_{14}\right)=z, \theta_{1}\left(c_{15}\right)=x$; if $y=1, z=x$, then the number of periodcycles of $\operatorname{ker} \theta_{1}$, that is 1 , equals $2(p-1) / 2$; so $p=2$; if $y=z=x$, it results again $p=2$; if $z=y \neq x$, then calling $r$ the order of $x y$, the number of period-cycles, 1 , is $2(p-1) / 2 r$, and so $r=p-1$, and the number of periods in the period-cycle, $2 k$, is $2 r$; so $r=k$. Since the number of proper periods, $g$, is $2(p-1) / 2$, we have $p=g+1, p=k+1, p=g+k-1$, and so $p=3$. If $y$ and $z$ have not the considered values, the kernel of $\theta_{1}$ has not signature (3).

Let now $\Gamma^{\prime}$ have signature (viii). If $\Gamma_{1}$ has signature (1), the epimorphism $\theta_{1}$ is given by $\theta_{1}\left(c_{10}\right)=x, \theta_{1}\left(c_{11}\right)=1, \theta_{1}\left(c_{12}\right)=1, \theta_{1}\left(c_{13}\right)=y, \theta_{1}\left(x_{1}\right)=z$, $\theta_{1}\left(e_{1}\right)=z^{-1}$. Then, if $y=z=1, G_{1}=Z_{2}$, and so $p=2$. If $y \neq 1 \neq z$, let $r$ be the order of $y z$. Then the number of period-cycles of the kernel, 1 , is $2(p-1) / 2 r$, and $r=p-1$; and the number of periods of the period-cycle, $2 k$, is $2 r$. So $r=k$, and $p=k+1$, impossible.

If $\Gamma_{1}$ has signature (2), the epimorphism $\theta_{1}$ satisfies $\theta_{1}\left(c_{10}\right)=x, \theta_{1}\left(c_{11}\right)=1$, $\theta_{1}\left(c_{12}\right)=y, \theta_{1}\left(c_{13}\right)=z, \theta_{1}\left(x_{1}\right)=t, \theta_{1}\left(e_{1}\right)=t^{-1}$. The number of proper periods, $p+1$, is $\beta(p-1)$, and so $p=2$, or 3 .

If $\Gamma_{1}$ has signature (3), then $\theta_{1}$ is given by $\theta_{1}\left(c_{10}\right)=x, \theta_{1}\left(c_{11}\right)=1, \theta_{1}\left(c_{12}\right)=1$, $\theta_{1}\left(c_{13}\right)=y, \theta_{1}\left(x_{1}\right)=1, \theta_{1}\left(e_{1}\right)=1$. If $y=z=1, G_{1}=Z_{2}$ and so $p=2$; if $y \neq 1 \neq z$, let $r$ be the order of $y z$, and then the number of period-cycles, 1 , is $2(p-1) / 2 r$, and $r=p-1$; the number of periods of the period-cycle, $2 k$, is $2 r$, so $r=k$; and the number of proper periods, $g$, is $2(p-1)$. Hence $p=2$.

When $\Gamma^{\prime}$ has signature (ix) and $\Gamma_{1}$ has signature (1), $\theta_{1}$ is given by $\theta_{1}\left(c_{10}\right)=x$, $\theta_{1}\left(c_{11}\right)=1, \theta_{1}\left(c_{12}\right)=1, \theta_{1}\left(c_{13}\right)=y, \theta_{1}\left(c_{14}\right)=x, x y$ having order 4 . Then $G_{1}=D_{4}$, and so $2(p-1)=8$; so $p=5, k=6$. Thus there exists $\theta: \Gamma^{\prime} \rightarrow G$, $|G|=16$, and ker $\theta$ has signature $(0,+,[-],\{(-),(-),(-),(-),(-),(-)\})$. As then 6 would have to divide 16 [7], this is impossible.

If $\Gamma_{1}$ has signature (2), $\theta_{1}$ is given by $\theta_{1}\left(c_{10}\right)=x, \theta_{1}\left(c_{11}\right)=1, \theta_{1}\left(c_{12}\right)=y$, $\theta_{1}\left(c_{13}\right)=z, \theta_{1}\left(c_{14}\right)=x$. Then the number of proper periods, $p+1$, is $2(p-1) / 4$ or $2(2(p-1) / 4)[2]$.

If $\Gamma_{1}$ has signature (3), the epimorphism is $\theta_{1}\left(c_{10}\right)=x, \theta_{1}\left(c_{11}\right)=1, \theta_{1}\left(c_{12}\right)=1$, $\theta_{1}\left(c_{13}\right)=y, \theta_{1}\left(c_{14}\right)=x$. Since $x y$ has order two, $1=2(p-1) / 4$, and so $p=3$.

If $\Gamma^{\prime}$ has signature (x), when $\Gamma_{1}$ has signature $(1)$, then $\theta_{1}\left(c_{10}\right)=1, \theta_{1}\left(c_{11}\right)=1$, $\theta_{1}\left(c_{12}\right)=1, \theta_{1}\left(x_{1}\right)=x, \theta_{1}\left(e_{1}\right)=x^{-1}$, with $x^{4}=1$. Hence $G_{1}=Z_{4}$, and $p=3$. 
When $\Gamma_{1}$ has signature (2), then $\theta_{1}\left(c_{10}\right)=x, \theta_{1}\left(c_{11}\right)=1, \theta_{1}\left(c_{12}\right)=y, \theta_{1}\left(x_{1}\right)=$ $z, \theta_{1}\left(e_{1}\right)=z^{-1}$, with $z^{2}=1$. Then the number of proper periods, $p+1$, is $2(p-1) / 2$, impossible.

$\Gamma_{1}$ having signature (3), we have $\theta_{1}\left(c_{10}\right)=1, \theta_{1}\left(c_{11}\right)=1, \theta_{1}\left(c_{12}\right)=1, \theta_{1}\left(x_{1}\right)=$ $x, \theta_{1}\left(e_{1}\right)=x^{-1}$. Then $G_{1}=Z_{2}$, and $p=2$.

When $\Gamma^{\prime}$ has signature (xi), $\Gamma_{1}$ must have signature (2) because it may not have nonempty period-cycles. In this case $\theta_{1}$ is defined by $\theta_{1}\left(x_{1}\right)=x, \theta_{1}\left(x_{2}\right)=y$, $\theta_{1}\left(e_{1}\right)=y^{-1} x^{-1}, \theta_{1}\left(c_{10}\right)=1$. The number of proper periods, $p+1$, is $\beta(p-1)$, $1 \leq \beta \leq 3$. Hence $p=2$ or 3 .

Finally, $\Gamma^{\prime}$ having signature (xii), one applies the same arguments of the last case of Lemma 2.

Thus the unique signature that we must consider is (i), $(0,+,[-],\{(2,2,2, n)\})$. Besides, $n>3$, since there are no surfaces of genus $p>3$ with $12(p-1)$ automorphisms.

In next theorems we obtain the group $G$ of automorphisms of $D / \Gamma$ we are looking for. Since $G=\Gamma^{\prime} / \Gamma$ and the surface is hyperelliptic, there exists $\Gamma_{1}$ such that $\Gamma \triangleleft \Gamma_{1} \triangleleft$ $\Gamma^{\prime}$. Hence there exist epimorphisms $\theta_{1}: \Gamma^{\prime} \rightarrow Z_{2}$ with $\operatorname{ker} \theta_{1}=G_{1}$ and $\theta: \Gamma^{\prime} \rightarrow Z_{2}$ with $\operatorname{ker} \theta=G$ and $\left|G_{1}\right|=\frac{1}{2}|G|$. The technique we use starts constructing $\theta_{1}$ and so determining conditions for $G_{1}$ : and from $G_{1}$ and the construction of $\theta$, conditions for $G$ are obtained. Finally the possible groups $G$ are checked.

THEOREM 1. Let $D / \Gamma$ be a hyperelliptic Klein surface of algebraic genus $p>3$, where $\Gamma$ has signature $(0,+,[-],\{(-), . k .,(-)\})$. Then, if $G=\Gamma^{\prime} / \Gamma$ is the automorphism group of $D / \Gamma$, having order at least $4(p-1), G=D_{p+1} \times Z_{2}$, and $\Gamma^{\prime}$ has signature $(0,+,[-],\{(2,2,2, p+1)\})$. Moreover, for each $p$, there exists a hyperelliptic Klein surface $D / \Gamma$ in the above conditions, having $D_{p+1} \times Z_{2}$ as its automorphism group.

PROOF. If $\Gamma_{1}$ is the group of hyperellipticity of $D / \Gamma, \Gamma_{1}$ has signature $(0,+,[-]$, $\{(2, .2 k, 2)\})$. If $G=\Gamma^{\prime} / \Gamma$ is the automorphism group of $D / \Gamma$, having order $\frac{4 n}{n-2}(p-1)$, there is an epimorphism $\theta_{1}: \Gamma^{\prime} \rightarrow G_{1}$, having kernel $\Gamma_{1}$, and $\left|G_{1}\right|=$ $\frac{2 n}{n-2}(p-1)$. The epimorphism $\theta_{1}$ is defined by $\theta_{1}\left(c_{10}\right)=x, \theta_{1}\left(c_{11}\right)=1, \theta_{1}\left(c_{12}\right)=1$, $\theta_{1}\left(c_{13}\right)=y, \theta_{1}\left(c_{14}\right)=x$. Since $\Gamma_{1}$ has no proper periods, the order of $x y$ is $n$. As $\Gamma_{1}$ has 1 period-cycle, we have $\frac{2 n}{n-2}(p-1) / 2 n=1$, and so $n=p+1$. So $G_{1}$ is generated by $x, y$, satisfying $x^{2}=y^{2}=(x y)^{p+1}=1$, and $G_{1}=D_{p+1}$.

Now we have an epimorphism $\theta$ from $\Gamma^{\prime}$ onto $G$ having kernel $\Gamma$. If $\alpha$ is a central element of $G$ and $\pi$ is the natural epimorphism from $G$ onto $G /\langle\alpha\rangle=G_{1}$, then $\theta_{1}$ must be equal to $\pi \theta$, since $D / \Gamma$ is a hyperelliptic surface.

As $|G|=4(p+1)$, then $\theta$ is defined by $\theta\left(c_{10}\right)=x$ or $x \alpha, \theta\left(c_{11}\right)=1$ or $\alpha$, $\theta\left(c_{12}\right)=1$ or $\alpha, \theta\left(c_{13}\right)=y$ or $y \alpha, \theta\left(c_{14}\right)=x$ or $x \alpha$, where $\theta\left(c_{10}\right)=\theta\left(c_{14}\right)$ and $\theta\left(c_{11}\right) \neq \theta\left(c_{12}\right)$; and $x^{2}=y^{2}=(x\langle\alpha\rangle y\langle\alpha\rangle)^{p+1}=1$. The group $G$ is generated by $x, y, \alpha$, and so $G=D_{p+1} \times Z_{2}$ or $D_{2(p+1)}$.

If $G=D_{p+1} \times Z_{2}$, for each $p$, let $\Gamma^{\prime}$ have signature $(0,+,[-],\{(2,2,2, p+1)\})$ and $\theta$ be the epimorphism from $\Gamma^{\prime}$ onto

$$
\left.D_{p+1} \times Z_{2}=\langle x, y, \alpha| x^{2}=y^{2}=\alpha^{2}=(x y)^{p+1}=1, \alpha \text { central }\right\rangle,
$$

given by $\theta\left(c_{10}\right)=x, \theta\left(c_{11}\right)=\alpha, \theta\left(c_{12}\right)=1, \theta\left(c_{13}\right)=y, \theta\left(c_{14}\right)=x$. The kernel of $\theta$ is orientable [8], and the signature of $\Gamma=\operatorname{ker} \theta$ is $\left(0,+,[-],\left\{(-), . k_{.},(-)\right\}\right)[\mathbf{3}, \mathbf{7}]$. 
This group $D_{p+1} \times Z_{2}$ is the full automorphism group of the surface, as it admits no other group of higher order.

If $G=D_{2(p+1)}$, there exists no epimorphism $\theta$ from $\Gamma^{\prime}$ onto $G$ since $\Gamma^{\prime}$ has no element of order $2(p+1)$.

Now we study the surfaces $D / \Gamma$ in which $\Gamma$ has signature $(g,+,[-],\{(-), . k .,(-)\})$.

THEOREM 2. Let $D / \Gamma$ be a hyperelliptic Klein surface of algebraic genus $p>3$, where $\Gamma$ has signature $\left(g,+,[-],\left\{(-), .{ }^{k},(-)\right\}\right), g>0,($ and then $k=1$ or 2$)$. Let $G=\Gamma^{\prime} / \Gamma$ be the automorphism group of $D / \Gamma$, and suppose that $G$ has order at least $4(p-1)$. Then $G=D_{p+1} \times Z_{2}$ (only for $p$ odd $)$ or $D_{2 p}($ for every $p)$ and $\Gamma^{\prime}$ has signature, respectively, $(0,+,[-],\{(2,2,2, p+1)\})$ and $(0,+,[-],\{(2,2,2,2 p)\})$. Moreover, for each $p$, there exist hyperelliptic Klein surfaces $D / \Gamma$ in each of the above conditions.

ProOF. Let $\Gamma_{1}$ be the group of the hyperellipticity of $D / \Gamma$. Then $\Gamma_{1}$ has signature $\left(0,+,\left[2,{ }^{2 g+k}, 2\right],\{(-)\}\right)$, and there exists an epimorphism $\theta_{1}$ from $\Gamma^{\prime}$ onto $G_{1}$ with kernel $\Gamma_{1}$. This epimorphism is defined by $\theta_{1}\left(c_{10}\right)=x, \theta_{1}\left(c_{11}\right)=1$, $\theta_{1}\left(c_{12}\right)=y, \theta_{1}\left(c_{13}\right)=z, \theta_{1}\left(c_{14}\right)=x$.

(a) If $x z$ has order $n$, since $\Gamma_{1}$ has proper periods, $y=z$. As $\Gamma_{1}$ has 1 periodcycle, $\frac{2 n}{n-2}(p-1) / 2 n=1$, and so $n=p+1$. Thus $G_{1}$ is generated by $x, y$, satisfying $x^{2}=y^{2}=(x y)^{p+1}=1$, and $G_{1}=D_{p+1}$.

The epimorphism $\theta$ from $\Gamma^{\prime}$ onto $G$ must again satisfy $\theta_{1}=\pi \theta, \pi$ being the epimorphism from $G$ onto $G /\langle\alpha\rangle$. The epimorphism $\theta$ is so defined by $\theta\left(c_{10}\right)=x$ or $x \alpha, \theta\left(c_{11}\right)=1, \theta\left(c_{12}\right)=y$ or $y \alpha, \theta\left(c_{13}\right)=y$ or $y \alpha, \theta\left(c_{14}\right)=x$ or $x \alpha$, where $\theta\left(c_{10}\right)=\theta\left(c_{14}\right), \theta\left(c_{12}\right) \neq \theta\left(c_{13}\right)$, and $x^{2}=y^{2}=(x\langle\alpha\rangle y\langle\alpha\rangle)^{p+1}=1$. Thus $G$ is generated by $x, y, \alpha$, and $G=D_{p+1} \times Z_{2}$ or $D_{2(p+1)}$.

Let $p+1$ be even. Then, if $G=D_{p+1} \times Z_{2}$, we take $\Gamma^{\prime}$ having signature $(0,+,[-],\{(2,2,2, p+1)\})$. The epimorphism $\theta$ from $\Gamma^{\prime}$ onto

$$
\left.D_{p+1} \times Z_{2}=\langle x, y, \alpha| x^{2}=y^{2}=\alpha^{2}=(x y)^{p+1}=1, \alpha \text { central }\right\rangle
$$

is given by $\theta\left(c_{10}\right)=x, \theta\left(c_{11}\right)=1, \theta\left(c_{12}\right)=y \alpha, \theta\left(c_{13}\right)=y, \theta\left(c_{14}\right)=x$. The kernel of $\theta$ is orientable [8] and the signature of $\Gamma=\operatorname{ker} \theta$ is $\left(\frac{p-1}{2},+,[-],\{(-),(-)\}\right)$.

If $G=D_{2(p+1)}$, there exists no epimorphism $\theta$ as $\Gamma^{\prime}$ has no elements of order $2(p+1)$.

In the valid case, $p+1$ even, $D_{p+1} \times Z_{2}$ is the full automorphism group of the surface.

(b) If $x z$ has order $n / 2$ and $y=z$, since $\Gamma_{1}$ has 1 period-cycle we have $\frac{2 n}{n-2}(p-1) n=1$, and so $p=n / 2$. Hence $G_{1}$ is generated by $x, y$, satisfying $x^{2}=y^{2}=(x y)^{p}=1$, and $G_{1}=D_{p}$.

The epimorphism $\theta$ from $\Gamma^{\prime}$ onto $G$ must be defined by $\theta\left(c_{10}\right)=x$ or $x \alpha, \theta\left(c_{11}\right)=$ $1, \theta\left(c_{12}\right)=y$ or $y \alpha, \theta\left(c_{13}\right)=y$ or $y \alpha, \theta\left(c_{14}\right)=x$ or $x \alpha$, where $\theta\left(c_{10}\right)=\theta\left(c_{14}\right)$, $\theta\left(c_{12}\right) \neq \theta\left(c_{13}\right)$, and $x^{2}=y^{2}=(x\langle\alpha\rangle y\langle\alpha\rangle)^{p}=1$. Thus $G$ is generated by $x, y, \alpha$, and $G$ is $D_{p} \times Z_{2}$ or $D_{2 p}$.

When $G=D_{p} \times Z_{2}$, for each $p$, let $\Gamma^{\prime}$ have signature $(0,+,[-],\{(2,2,2,2 p)\})$ and $\theta$ be the epimorphism from $\Gamma^{\prime}$ onto $D_{p} \times Z_{2}$. If $p$ is even, the group $D_{p} \times Z_{2}$ has no elements of order $2 p$, and so $\theta$ does not exist.

If $G=D_{2 p}=\left\langle x^{\prime}, y^{\prime} \mid x^{\prime 2}=y^{\prime 2}=\left(x^{\prime} y^{\prime}\right)^{2 p}=1\right\rangle$, and $\theta$ is given by $\theta\left(c_{10}\right)=x^{\prime}$, $\theta\left(c_{11}\right)=1, \theta\left(c_{12}\right)=y^{\prime}\left(x^{\prime} y^{\prime}\right)^{p}, \theta\left(c_{13}\right)=y^{\prime}, \theta\left(c_{14}\right)=x^{\prime}$, the kernel of $\theta$ is orientable 
$[8]$ and $\Gamma=\operatorname{ker} \theta$ has signature $\left(\frac{p-1}{2},+,[-],\{(-),(-)\}\right)(p$ odd $)$ or $\left(\frac{p}{2},+,[-],\{(-)\}\right)$ ( $p$ even). The group $D_{2 p}$ is the full automorphism group of the surface.

(c) If $x z$ has order $n / 2$ and $y \neq z$, the number of proper periods of $\Gamma_{1}$ is $p+1=$ $\frac{2 n}{n-2}(p-1) / n=\frac{2}{n-2}(p-1)$, and so $p=n /(4-n)$, impossible since $n \geq 1$.

THEOREM 3. Let $D / \Gamma$ be a hyperelliptic Klein surface of algebraic genus $p>3$, where $\Gamma$ has signature $(g,-,[-],\{(-), . k .,(-)\})$. Then if $G=\Gamma^{\prime} / \Gamma$ is the automorphism group of $D / \Gamma$, having order at least $4(p-1)$, we have $g=1$ (and so $k=p$ ) and $G=D_{2 p}, \Gamma^{\prime}$ having signature $(0,+,[-],\{(2,2,2,2 p)\})$. Moreover, for each $p$, there exists a hyperelliptic Klein surface $D / \Gamma$ in the above conditions.

PROOF. Let $\Gamma_{1}$ be the group of the hyperellipticity of $D / \Gamma$. Then $\Gamma_{1}$ has signature $(0,+,[2, . \stackrel{g}{.}, 2],\{(2, .2 k ., 2)\})$ and there exists $\theta_{1}$ epimorphism from $\Gamma^{\prime}$ onto $G_{1}$ with kernel $\Gamma_{1}$. This epimorphism is defined by $\theta_{1}\left(c_{10}\right)=x, \theta_{1}\left(c_{11}\right)=1$, $\theta_{1}\left(c_{12}\right)=1, \theta_{1}\left(c_{13}\right)=y, \theta_{1}\left(c_{14}\right)=x$. In this situation either $x=y$ or $x y$ has order $n / 2$. If $x=y$, the group $G_{1}=Z_{2}$ and $p<2$, impossible. Hence $x \neq y$, $(x y)^{n / 2}=1$. Since $\Gamma_{1}$ has 1 period-cycle, $1=\frac{2 n}{n-2}(p-1) / n$ and so $p=n / 2$. Since the period-cycle has $2 k$ periods, it is $2 k=n$, and $k=n / 2$.

$G_{1}$ is so generated by $x, y$, satisfying $x^{2}=y^{2}=(x y)^{p}=1$, and $G_{1}=D_{p}$. The epimorphism $\theta$ from $\Gamma^{\prime}$ onto $G$ with kernel $\Gamma$ satisfies $\theta_{1}=\pi \theta$ where $\pi$ is the epimorphism from $G$ onto $G /\langle\alpha\rangle$. Hence $\theta$ is defined by $\theta\left(c_{10}\right)=x$ or $x \alpha, \theta\left(c_{11}\right)=1$ or $\alpha, \theta\left(c_{12}\right)=1$ or $\alpha, \theta\left(c_{13}\right)=y$ or $y \alpha, \theta\left(c_{14}\right)=x$ or $x \alpha$, with $\theta\left(c_{10}\right)=\theta\left(c_{14}\right)$, $\theta\left(c_{11}\right) \neq \theta\left(c_{12}\right), x^{2}=y^{2}=(x\langle\alpha\rangle y\langle\alpha\rangle)^{p}=1$. Thus $G$ is generated by $x, y, \alpha$, and $G=D_{p} \times Z_{2}$ or $D_{2 p}$.

When $G=D_{p} \times Z_{2}$, for each $p$, let $\Gamma^{\prime}$ have signature $(0,+,[-],\{(2,2,2,2 p)\})$ and $\theta$ be the epimorphism from $\Gamma^{\prime}$ onto $D_{p} \times Z_{2}$. If $p$ is even, $G$ has not elements of order $2 p$, and $\theta$ does not exist.

If $G=D_{2 p}=\left\langle x^{\prime}, y^{\prime} \mid x^{\prime 2}=y^{\prime 2}=\left(x^{\prime} y^{\prime}\right)^{2 p}=1\right\rangle, \theta$ is given by $\theta\left(c_{10}\right)=x^{\prime}, \theta\left(c_{11}\right)=$ $1, \theta\left(c_{12}\right)=\left(x^{\prime} y^{\prime}\right)^{p}, \theta\left(c_{13}\right)=y^{\prime}, \theta\left(c_{14}\right)=x^{\prime}$. The kernel of $\theta$ is nonorientable [8] and its signature is $(1,-,[-],\{(-), . \stackrel{p}{.},(-)\})$. The group $D_{2 p}$ is the full group of automorphisms of the surface.

These results may be summarized in the following table:

\begin{tabular}{|c|c|c|c|}
\hline & $\begin{array}{l}\text { Hyperelliptic surface } D / \Gamma \\
\text { Signature of } \Gamma(p>3)\end{array}$ & Signature of $\Gamma^{\prime}$ & Group $G=\Gamma^{\prime} / \Gamma$ \\
\hline (i) & $(0,+,[-],\{(-), \stackrel{p-1}{*},(-)\})$ & $(0,+,[-],\{(2,2,2, p+1)\})$ & $D_{p+1} \times Z_{2}$ \\
\hline (ii) & $\left(\frac{p}{2},+,[-],\{(-)\}\right)$ & $(0,+,[-],\{(2,2,2,2 p)\})$ & $D_{2 p}$ \\
\hline (iii) & $\left(\frac{p-1}{2},+,[-],\{(-),(-)\}\right)$ & $\begin{array}{l}(0,+,[-],\{(2,2,2, p+1)\}) \\
(0,+,[-],\{(2,2,2,2 p)\})\end{array}$ & $\begin{array}{l}D_{p+1} \times Z_{2} \\
D_{2 p}\end{array}$ \\
\hline (iv) & $(1,-,[-],\{(-), . \stackrel{p}{.},(-)\})$ & $(0,+,[-],\{(2,2,2,2 p)\})$ & $\overline{D_{2 p}}$ \\
\hline
\end{tabular}

Observe that nonorientable hyperelliptic Klein surfaces of genus $p>3$ and topological genus $g \geq 2$ have always less than $4(p-1)$ automorphisms.

REMARKS. (1) Observe that May obtains in [10, pp. 275, 279] examples of Klein surfaces with the topological type and the automorphism group that appear in cases (i) and (iv) of the above table.

(2) Also in [10, Theorems 2,3] it was proven that infinite values of $p$ verify that the order of the automorphism group of an orientable (nonorientable) surface of 
genus $p$ is at most $4(p+1)(4 p)$. Observe that if the surface is hyperelliptic, this upper bound is obtained for all values of $p$.

Observe too that the table gives the upper bound for the order of the automorphism group of a hyperelliptic surface, according to its topological type.

(3) May proved in [11] that the upper bound of the order of an automorphism of a Klein surface of genus $p$ is $2(p+1)$ for orientable surfaces of even $p$, and $2 p$ otherwise. We show in the above table families of hyperelliptic surfaces attaining these bounds, coinciding in some cases with the examples of [11].

4. Real algebraic curves. All results on bordered Klein surfaces can be stated in terms of real algebraic curves, since given a Klein surface of algebraic genus $p$ with $k$ boundary components, there exists a real projective irreducible curve of genus $p$ with $k$ connected components, whose group of birational isomorphisms coincides with the automorphism group of the surface, and conversely. The surface is orientable if and only if the curve disconnects its complexification.

Consequently, this equivalence allows us to determine all hyperelliptic real curves of genus $p>3$ with at least $4(p-1)$ birational isomorphisms, by a simple translation of the above results.

The authors wish to thank the referee for his helpful suggestions.

\section{REFERENCES}

1. N. I. Alling and N. Greenleaf, Foundations of the theory of Klein surfaces, Lecture Notes in Math., vol. 219, Springer-Verlag, Berlin-Heidelberg-New York, 1971.

2. E. Bujalance, Proper periods of normal NEC subgroups with even index, Rev. Mat. Hisp.-Amer. (4) 41 (1981), 121-127.

3. E. Bujalance and J. J. Etayo, Hyperelliptic Klein surfaces with maximal symmetry, Proc. Warwick and Durham Symposia 1984, London Math. Soc. Lecture Note Series, 112, 1986, pp. 289-296.

4. E. Bujalance, J. J. Etayo, and J. M. Gamboa, Hyperelliptic Klein surfaces, Quart. J. Math. Oxford Ser. (2) 36 1985, pp. 141-157.

5. _ Groups of automorphisms of hyperelliptic Klein surfaces of genus three, Michigan Math. J. 33 (1986), 55-74.

6. E. Bujalance and J. M. Gamboa, Automorphisms groups of algebraic curves of $\mathbf{R}^{n}$ of genus 2, Arch. Math. 42 (1984), 229-237.

7. J. A. Bujalance, Normal subgroups of even index of an NEC group, Arch. Math. (to appear).

8. A. H. M. Hoare and D. Singerman, The orientability of subgroups of plane groups, London Math. Soc. Lecture Note Series, 71, 1982, pp. 221-227.

9. C. L. May, Automorphisms of compact Klein surfaces with boundary, Pacific J. Math. 59 (1975), 199-210.

10. $A$ bound for the number of automorphisms of a compact Klein surface with boundary, Proc. Amer. Math. Soc. 63 (1977), 273-280.

11. __ Cyclic automorphism groups of compact bordered Klein surfaces, Houston J. Math. 3 (1977), 395-405.

Departamento de Matemática Fundamental, Facultad de Ciencias, U.N.E.D., 28040 MADRID-SPAIN

Departamento de Geometría y Topología, Facultad de Ciencias MatemáTICAS, UNIVERSIDAD COMPLUTENSE, 28040 MADRID-SPAIN 